\title{
Pneumopericardium after Pericardiocentesis: An Underlying Tracheopericardial Fistula
}

\author{
Mohammed Elzeneini ${ }^{1}$, Jared Freitas ${ }^{2}$, and Seyed Aalaei-Andabili ${ }^{3}$ \\ ${ }^{1}$ University of Florida \\ ${ }^{2}$ Vanderbilt University \\ ${ }^{3}$ University of Maryland School of Medicine
}

July 25, 2020

\begin{abstract}
Pneumopericardium is a rare entity in adults. We present a case of non-iatrogenic pneumopericardium that manifested after a pericardiocentesis procedure for pericardial effusion, resulting from an underlying tracheopericardial fistula. We discuss our patient's presentation and management of this unique entity.
\end{abstract}

\section{Key Clinical Message}

Accumulation of air in the pericardium after pericardiocentesis may indicate a malignancy-related fistulous communication with the trachea, bronchi or esophagus.

\section{Introduction}

Pneumopericardium, the presence of air in the pericardial cavity, is a rare entity in adults that is typically described as a result of chest trauma or iatrogenic complication of an invasive procedure. We present a case of non-iatrogenic pneumopericardium manifesting after pericardiocentesis, resulting from an underlying fistulous communication between the distal trachea and pericardial cavity, as a complication of a malignant glomus tumor of the trachea.

\section{Case Presentation}

A 62-year-old man with a history of malignant glomus tumor of the trachea status post distal tracheal stenting and salvage chemotherapy presented to the emergency room with chest pain. Initial work-up revealed a pneumonia and new-onset atrial fibrillation for which he was admitted for management. Transthoracic echocardiography showed a large circumferential pericardial effusion without tamponade physiology. A percutaneous pericardiocentesis was done with removal of $400 \mathrm{ml}$ of pericardial fluid, of which fluid analysis showed an exudate with positive cultures. The patient was treated with appropriate intravenous antibiotics and the pericardial drain was removed 48 hours later. A follow-up chest computed tomography (CT) showed air in the mediastinum and pericardium (Figure 1, panel A) and visualized a tracheopericardial fistula (Figure 1, panel B) resulting from anterior distal tracheal wall ulceration by the tracheal malignancy. After discussion with the patient, a bronchoscopy was performed by the interventional pulmonary team for palliative removal of the tracheal mass and re-stenting of the trachea to block the fistulous connection. The patient continued to stabilize as spontaneous absorption of the air occurred and the infection was treated, and was eventually discharged home with palliative care.

\section{Discussion}


Pneumopericardium is rare in adults and is typically described as a result of chest trauma or iatrogenic complication of an invasive procedure (1)(2). It has also been described spontaneously (1) and as a rare complication of pericardiocentesis (3)(4). A recent case series brought light to cancer-related pneumopericadium, and identified 11 reported cases in the literature, 10 of which were in adult males and occurred as a result of a fistulous communication with the esophagus or bronchus (5). To our knowledge, this is the first reported case of pneumopericardium as a result of a fistulous communication with the distal trachea, in this case, manifesting after pericardiocentesis. Fistulous pneumopericardium should be suspected whenever a patient with gastroesophageal or tracheobronchial malignancy presents with chest pain or shortness of breath. Diagnosis can be achieved by chest X-ray showing the appearance of a radiolucent rim around the cardiac shadow, and computed tomography can help demonstrate the presence of a fistulous communication. Treatment depends on the hemodynamic stability of the patient, the presence and location of a fistula, and the overall prognosis and functional status of the patient. Patients with hemodynamic compromise should be treated with pericardiocentesis, while hemodynamically stable patients can be managed conservatively with watchful surveillance for spontaneous absorption of air. If a fistula is present, stenting using endoscopy or bronchoscopy can be pursued to block the fistulous connection and prevent further accumulation of pericardial air. In the presence of an infiltrating malignancy, goals of care should be discussed with the patient and family.

\section{Conclusion}

Pneumopericardium in adults can result from a fistulous communication between the bronchus, trachea or esophagus and the pericardial cavity. To our knowledge, this is the first reported case of a communication from the trachea. Our patient was successfully managed with tracheal stenting.

\section{Author Contribution}

Mohammed Elzeneini designed and drafted the manuscript. Jared Freitas shared in drafting the manuscript. Seyed Hossein Aalaei-Andabili critically revised the manuscript.

\section{Conflict of Interest Statement}

None of the authors have any no conflicts of interest to declare.

\section{References}

1. Brander L, Ramsay D, Dreier D, Peter M, Graeni R. Continuous left hemidiaphragm sign revisited: a case of spontaneous pneumopericardium and literature review. Heart. 2002 Oct ;88(4):e5.

2. Green PG, Herring N. Pneumopericardium and Pneumomediastinum After Implantation of a Cardiac Resynchronization Pacemaker. JACC Case Rep. 2019 Oct;1(3):381-384.

3. Narins CR, Lee J, Cole M, Ling FS. Pneumopericardium Following Pericardiocentesis. Am J Med. 2016 Sep 1;129(9):e181-182.

4. Wakabayashi Y, Hayashi T, Mitsuhashi T, Fujita H. Tension pneumopericardium after pericardiocentesis: Useful echocardiographic obscured heart sign and effective postural change during air aspiration. Heart Rhythm. 2018 Jul 1;15(7):1116.

5. Hirani S, Velez Martinez CS, Patan S, Kavanaugh M. Cancer-Related Pneumopericardium: A Case Report and Literature Review. Case Rep Oncol. 2020 Jan 20;13(1):23-28.

\section{Figure Legend}

Figure 1. Chest computed tomography showing air in the pericardium and mediastinum (panel A) and a tracheopericardial fistula from the distal trachea, with a patent tracheal stent (panel B). 


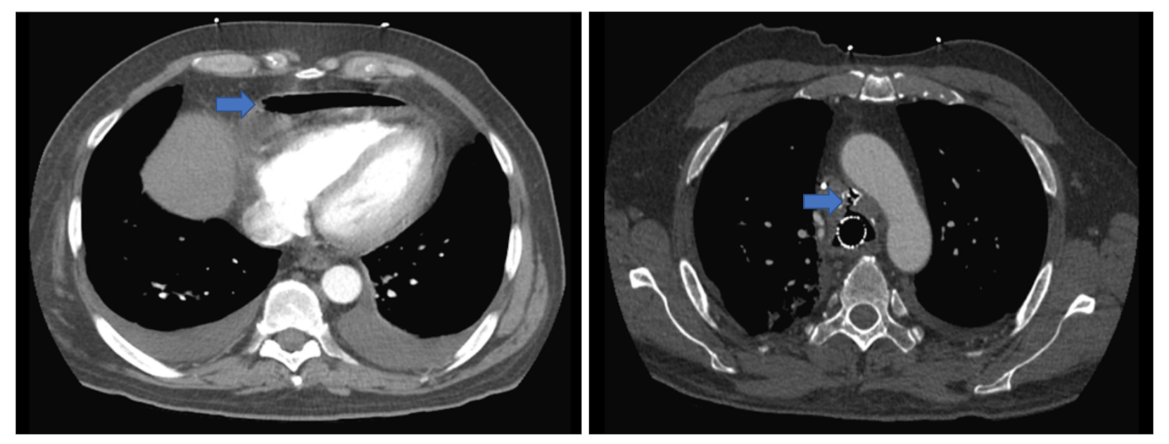

